\title{
Factores de riesgo asociados a hígado graso de origen no alcohólico en una población del Caribe Colombiano
}

\section{Risk Factors Associated with Nonalcoholic Fatty Liver Disease in a Colombian Caribbean Population}

Lina Lambis A., ${ }^{1}$ José Belisario Solana T., MD, ${ }^{2}$ Bertha Gastelbondo P., ${ }^{1}$ Daniel Romero S., ${ }^{1}$ Daniela Garrido C., ${ }^{1}$ Walter Puello R., MD³ María C. García E., ${ }^{1}$ Amileth Suarez C. ${ }^{4}$

Grupo Prometeus \& Biomedicina aplicada a las Ciencias clínicas. Laboratorio de Bioquímica, Facultad de Medicina, Universidad de Cartagena. Cartagena, Colombia.

2 Médico Unidad de Hígado Vías biliares, Hospital Universitario del Caribe. Cartagena, Colombia.

3 Residente de Cirugía, Facultad de Medicina, Universidad de Cartagena. Cartagena, Colombia.

${ }^{4}$ Directora del grupo Prometeus \& Biomedicina aplicada a las ciencias clínicas. Docente de Bioquímica (PhD), Facultad de Medicina, Universidad de Cartagena, Colombia

Contacto: asuarezc1@unicartagena.edu.co

Fecha recibido: $28-05-15$

Fecha aceptado: $18-04-16$

\begin{abstract}
Resumen
La enfermedad hepática grasa no alcohólica NAFLD (Non-alcoholic fatty liver disease) es un problema de salud, así lo demuestran las diversas investigaciones alrededor del mundo, donde se evidencia una alta prevalencia de la patología. El objetivo del estudio piloto fue describir en términos de variables demográficas, epidemiológicas y bioquímicas la relación de factores de riesgo asociados a NAFLD de una muestra de pacientes que acudieron a la sección de Hígado y vías biliares del Hospital Universitario del Caribe (Cartagena, Colombia). El estudio piloto fue descriptivo prospectivo, fueron revisadas historias clínicas para análisis de variables epidemiológicas-demográficas y valorados por medición espectrofotométrica la actividad enzimática de transaminasas para la relación De Ritis (AST/ALT), niveles de colesterol y triglicéridos. Los resultados sugieren que la población subdividida en obesos, diabetes mellitus, hipertensos/obesos, diabetes/obesos y principalmente del género femenino presenta mayor riesgo de padecer hígado graso, con lo que amerita un estudio bioquímico molecular y un diagnóstico definitivo de NAFLD en la población de estudio.
\end{abstract}

Palabras clave

Hígado graso, NAFLD, gastroenterología.

\section{Abstract}

Nonalcoholic fatty liver disease (NAFLD) is a health problem that has been demonstrated by research from everywhere around the world where the disease's prevalence appears to be high. The aim of this pilot study was to describe the relationship of risk factors associated with NAFLD in terms of demographic, epidemiological and biochemical variables. Patients included in the study were drawn from people who came to the liver and biliary tract section of the Hospital Universitario del Caribe in Cartagena, Colombia. This was a prospective and descriptive pilot study that reviewed medical records for analysis of epidemiological and demographic variables. It assessed spectrophotometric measurement of transaminases for determination of De Ritis ratios (AST/ALT), and relationship to levels of cholesterol and triglycerides. The results suggest that risks of developing NAFLD were higher among women, among patients who were obese, among patients who had diabetes mellitus, among patients who had hypertension and obesity, and among patients who had diabetes combined with obesity. Patients at risk merit biochemical molecular study and a definitive diagnosis.

\section{Keywords}

Fatty liver, NAFLD, gastroenterology. 


\section{INTRODUCCIÓN}

La enfermedad hepática grasa no alcohólica NAFLD (Nonalcoholic fatty liver disease, hígado graso o esteatosis hepática no alcohólica) es una condición clínico-patológica caracterizada por la infiltración de grasa en los hepatocitos del parénquima hepático en un porcentaje que excede del $5 \%$ al $10 \%$ de peso y anormalidad persistente de las enzimas hepáticas, además del consumo de alcohol no significativo. También es considerada la manifestación hepática del síndrome metabólico $(1,2)$. La prevalencia de NAFLD está relacionada con la obesidad y resistencia insulina; varía dependiendo del área geográfica (Tabla 1), pero se puede estimar un rango entre $20 \%-30 \%$ a nivel mundial, principalmente en países industrializados del mundo occidental (3).

Tabla 1. Prevalencia de NAFLD según el área geográfica.

\begin{tabular}{lccc}
\hline $\begin{array}{c}\text { Zona } \\
\text { geográfica }\end{array}$ & Referencia & Población estudiada & $\begin{array}{c}\text { Prevalencia } \\
\text { NAFLD }\end{array}$ \\
\hline Nigeria & $(4)$ & General & $8,7 \%$ \\
Japón & $(5)$ & General & $29,7 \%$ \\
India & $(6)$ & General & $9 \%$ \\
Italia & $(7)$ & General & $58 \%$ \\
& $(8)$ & General & $25,5 \%$ \\
España & $(9)$ & General & $25,8 \%$ \\
Ucrania & $(10)$ & Diabetes tipo II & $46,2 \%$ \\
EE. UU. & $(11)$ & General & $45 \%$ \\
México & $(12)$ & Síndrome metabólico & $82,9 \%$ \\
Brasil & $(13)$ & General & $33,5 \%$ \\
Chile & $(14)$ & Obesos & $63 \%$ \\
\hline
\end{tabular}

Clínicamente, la NAFLD presenta un amplio espectro patológico que incluye una simple esteatosis macrovesicular, hígado graso acompañado por inflamación, fibrogénesis y necrosis hepatocelular (esteatosis hepática no alcohólica), lo que aumenta el riesgo a fibrosis perdiendo la función del hígado; en algunos casos desarrollo del hepatocarcinoma (15-20). Entre los factores de riesgo asociados a esta patología están estilos de vida como dieta y ejercicio, resistencia a insulina, diabetes tipo II, dislipidemia, edad, género y factores genéticos (21). Es de resaltar que la obesidad y la resistencia a la insulina se consideran los factores de riesgo asociados más frecuentemente a la NAFLD; así, el grado de esteatosis puede ser correlacionado con índice de masa corporal (IMC) y la diabetes puede ser un factor independiente de predictor de NAFLD o cualquier daño hepático.

El mecanismo bioquímico es complejo e incluye acumulo de triglicéridos, resistencia a insulina, desregulación del metabolismo de lípidos, estrés oxidativo por disfunción mitocondrial y peroxidación lipídica, así como disfunción de respuesta inmune innata. Estos procesos se encuentran asociados con múltiples polimorfismos en genes, que están vinculados con esos procesos $(22,23)$. Por tanto, la genética puede ser un determinante de riesgo y severidad del desarrollo de hígado graso (24). Para el diagnóstico, las pruebas bioquímicas en particular las transaminasas, triglicéridos y colesterol son pruebas sensibles para la esteatosis simple, pero se requiere confirmar con imágenes histológicas o pruebas de imagenología (ultrasonografía, tomografía computarizada, resonancia magnética, fibroscan, etc.).

Uno de los parámetros que ha estado en el olvido, pero que su utilidad fue demostrada en 1957 por Fernando De Ritis, es la relación AST/ALT (aspartato-aminotransferasa (EC 2.6.1.1)/alanina-aminotransferasa (EC 2.6.1.2) (25), el cual predice daño hepatocelular. La ALT está presente solo en el citoplasma, mientras que la AST presenta dos isoformas, la citoplasmática (cAST) y la mitocondrial (mAST); estas últimas son inmunológicamente distintas, la mAST es la isoenzima más prevalente, con aproximadamente el 80\% del total de actividad en hígado humano (26). La actividad de estas transaminasas es reflejo de la actividad metabólica en los tejidos en los cuales se expresan, y representan la relación importante entre el metabolismo de carbohidratos y proteínas (Figuras 1 y 2). La relación AST/ ALT (De Ritis ratio) es un valor útil de apoyo para predecir un indicio muy probable de daño hepático que tiende a cirrosis, debido a que cuando hay muerte hepatocelular se incrementan los niveles séricos de AST, comparado con ALT, y refleja el daño de la proporción celular siempre que AST sea muy superior a ALT. Así, relaciones superiores a 2,0 reflejan liberación intracelular de la enzima (mAST y cAST) y por tanto daño hepatocelular.

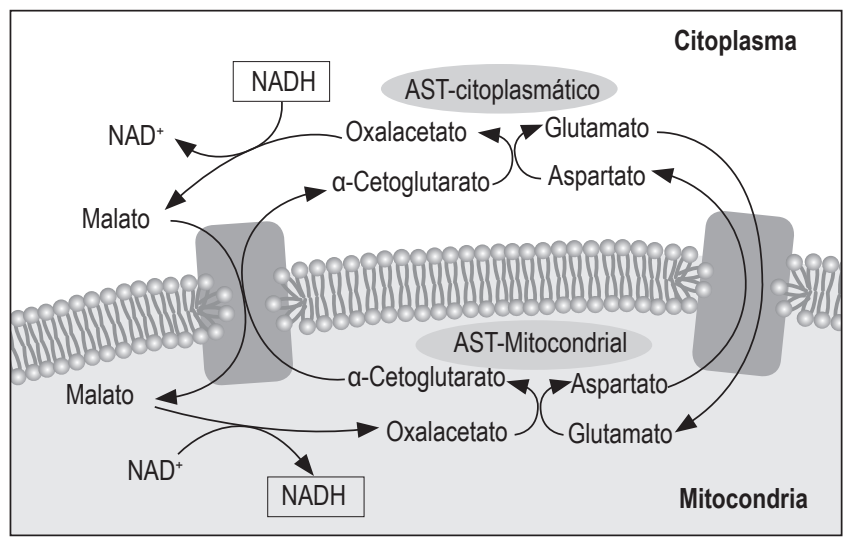

Figura 1. Función de las isoformas de AST en el citoplasma y mitocondria. Adaptado de Botros M, 2013 (27).

El objetivo de esta primera parte del estudio piloto fue valorar en términos demográficos, epidemiológicos y bioquímicos la relación de factores de riesgo asociados que 
conducen a NAFLD; en los pacientes que acudieron a la sección de Hígado y vías biliares del Hospital Universitario del Caribe, Cartagena.

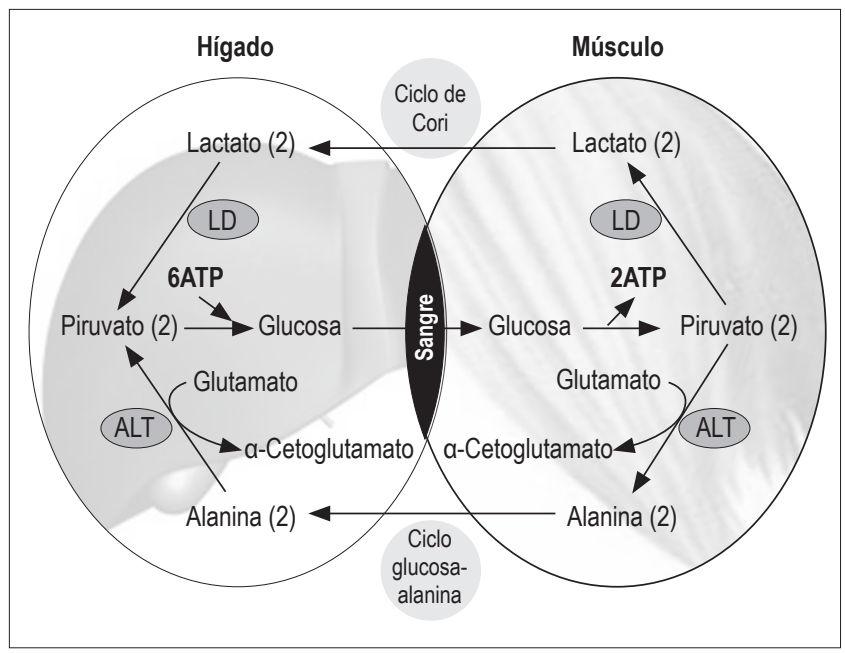

Figura 2. Función de ALT en el ciclo de glucosa-alanina. Adaptado de Botros M, 2013 (27).

\section{METODOLOGÍA}

En este estudio piloto descriptivo prospectivo fueron incluidos 105 pacientes adultos con posibles factores de riesgo asociados a hígado graso que acudieron a la sección de cirugía de hígado y vías biliares del hospital Universitario del Caribe, durante el período de septiembre de 2014 abril de 2015. Los criterios de exclusión fueron: consumo de alcohol (20 g/día para hombres y $10 \mathrm{~g}$ /día para mujeres), hepatitis viral, medicamentos hepatotóxicos, hemocromatosis y enfermedad de Wilson. Fueron determinadas las características demográficas, el motivo de remisión del paciente, los antecedentes referidos en la historia clínica, los datos del examen físico y las pruebas de laboratorio. Para el análisis estadístico se analizó la información mediante estadística descriptiva, se calcularon medidas de tendencia central, porcentajes y frecuencias absolutas; se tomó un error del 5\%. Se utilizó Graphpad prism para el análisis de los datos. Todos los datos de este estudio fueron conducidos de acuerdo con los principios declarados en la XVIII Asamblea Médica Mundial (Helsinki, 1964).

\section{Análisis bioquímico}

La muestra de sangre fue obtenida por punción venosa, se recolectó en tubos que contenían EDTA como anticoagulante; para obtención del plasma las muestras se centrifugaron por 10 minutos a 2500 revoluciones por minuto (rpm) a $4{ }^{\circ} \mathrm{C}$. Los ensayos se realizaron en el Laboratorio de Investigación de Bioquímica de la Facultad de Medicina.
La determinación de actividad enzimática de transaminasas, triglicéridos y colesterol se realizó según indicaciones del fabricante (Biosystems). Los valores de referencia establecidos para valorar actividad enzimática de AST y ALT, colesterol y triglicéridos fueron 40 y $41 \mathrm{U} / \mathrm{L}, 200 \mathrm{mg} / \mathrm{dL}$, $150 \mathrm{mg} / \mathrm{dL}$ respectivamente. La actividad enzimática (U) fue establecida como concentración de enzima que cataliza la conversión de $1 \mu \mathrm{mol}$ de sustrato en un minuto. Todas las determinaciones se realizaron por triplicado en el equipo Tecan infinite M200.

\section{RESULTADOS}

En este estudio fueron tomadas 105 historias clínicas para el análisis descriptivo de algunos factores de riesgo que conducen a hígado graso, que corresponden a pacientes que ingresan a la Unidad de hígado y vías biliares del Hospital Universitario del Caribe. El rango de edad de los pacientes incluidos en el estudio fue jóvenes (18-23 años), adultos (24-59 años) y adulto mayor (mayores de 60 años). Los resultados preliminares muestran que la población mayoritaria está representada por individuos adultos con un $52 \%$. Aun así, es preocupante el $18 \%$ que representa la población joven del estudio. Por otra parte, el $85 \%$ de los individuos del estudio corresponde al género femenino y demográficamente el $82 \%$ de toda la población vive en área urbana (Tabla 2). Dentro de los factores de riesgo asociados a hígado graso no alcohólico se encontraron diabetes

Tabla 2. Características de la población de estudio

\begin{tabular}{lcc}
\hline \multicolumn{1}{c}{ Total muestras ( $\mathrm{n}=\mathbf{1 0 5})$} & & \\
\hline $\begin{array}{l}\text { Edad, media (desviación estándar) } \\
\text { Género }\end{array}$ & 51,3 & $(16,9)$ \\
$\quad$ Femenino, $\mathrm{n}(\%)$ & & \\
$\quad$ Masculino, $\mathrm{n}(\%)$ & 85 & $(81,0)$ \\
Procedencia & 20 & $(19,0)$ \\
$\quad$ Urbano, $\mathrm{n}(\%)$ & & \\
$\quad$ Rural, $\mathrm{n}(\%)$ & 63 & $(60,3)$ \\
Clínica & 42 & $(39,7)$ \\
$\quad$ Obesidad, $\mathrm{n}(\%)$ & & \\
$\quad$ Diabetes, $\mathrm{n}(\%)$ & 29 & $(28,0)$ \\
$\quad$ HT/Obeso, $\mathrm{n}(\%)$ & 21 & $(20,0)$ \\
$\quad$ DM/HT, $\mathrm{n}(\%)$ & 18 & $(17,0)$ \\
Antropométrico & 37 & $(35,0)$ \\
$\quad$ Peso, media (desviación estándar) & & \\
$\quad$ Talla, media (desviación estándar) & 74,8 & $(13,6)$ \\
$\quad$ IMC, media (desviación estándar) & 1,60 & $(0,08)$ \\
\hline
\end{tabular}


mellitus tipo II (DM), obesidad e hipertensión arterial (HT). Se observó una frecuencia de igual importancia para todas las condiciones, fue $28 \%$ para obesidad, $20 \%$ DM y $38 \%$ para hipertensión arterial; se tomaron valores absolutos sin combinar las patologías. La Figura 3 muestra el porcentaje y asocia grupos con combinación de dos patologías, la distribución queda así: $28 \%$ a los pacientes obesos, $20 \%$ DM, 17\% HT/obeso y 35\% DM/HT.

Con respecto a los marcadores bioquímicos, para la relación de Ritis (AST/ALT) se obtuvo un valor significativamente superior a $1,0(p<0,05)$, triglicéridos por encima de $160 \mathrm{mg} / \mathrm{dL}$ y colesterol menor de $200 \mathrm{mg} / \mathrm{dL}$ en todos los factores asociados a hígado graso abordados. La población de pacientes incluidos en el estudio muestra, según las características de la Tabla 3, que la población adulta caracterizada como HT/obeso presenta mayor índice De Ritis y un promedio de índice de masa corporal de 36,6.

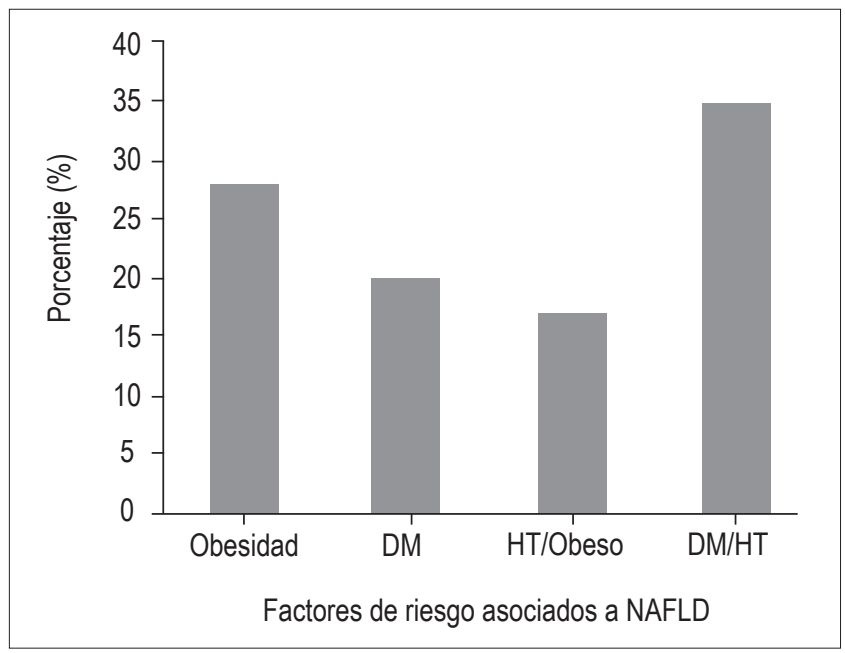

Figura 3. Porcentaje de las frecuencias de las patologías asociadas a NAFLD. Diabetes mellitus (DM), hipertensión (HT).

\section{DISCUSIÓN}

La enfermedad de hígado graso es extensamente estudiada a nivel mundial por ser un problema de salud pública importante. La incidencia se ha estimado en uno de cada tres adultos en países desarrollados y puede afectar a los individuos a cualquier edad. Se ha demostrado una prevalencia importante en un estudio prospectivo de cohorte en pacientes adultos asintomáticos usando ultrasonido (US) y biopsia hepática, en el cual se encontró una prevalencia de NAFLD de $46 \%(28,29)$. La etnia también influye en la prevalencia de NAFLD (30). Así, la población de América del Sur ha sido registrada con el mayor número de individuos con esta patología a nivel mundial $(31,32)$. De acuerdo con los resultados, los principales factores de riesgo asociados a NAFLD en la población son: obesidad, DM, HT, índice Ritis $>1,0$ y dislipidemia; así como se reporta en las guías de práctica clínica para NAFLD (Evidence-based clinical practice guidelines for nonalcoholic fatty liver disease/nonalcoholic steatohepatitis), con lo cual amerita la realización de un estudio bioquímico-molecular amplio, que incluya diagnóstico de NAFLD para mayor comprensión de esta patología (33).

Si bien ha sido reportada una relación lineal entre la edad y la frecuencia de los factores asociados y confirmados de NAFLD, pero en nuestro estudio no fue así. Dos posibles razones podrían ser la muerte temprana por enfermedad hepática y la baja cobertura de salud de la población. Por otra parte, el hecho de que el $18 \%$ de los pacientes con enfermedad hepática de hígado graso sean individuos jóvenes, es un dato inquietante que repercutiría en la historia natural de esta patología, considerando que su hígado estaría expuesto durante un mayor tiempo a alteraciones metabólicas, que finalmente podrían llevar a un incremento del riesgo de progresión y complicación de esta patología.

Tabla 3. Características clínicas de la población con factores de riesgo asociados a NAFLD

\begin{tabular}{lcccc}
\hline & Obesidad & Diabetes mellitus & Obesidad/HTA & Diabetes/HTA \\
& $\bar{X}(D E)$ & $\bar{X}(D E)$ & $\bar{X}(D E)$ & $67(14,1)$ \\
\hline Edad & $49,9(17,0)$ & $61,8(13,8)$ & $64,8(21,3)$ & $30,6(4,5)$ \\
IMC $\left(\mathrm{kg} / \mathrm{m}^{2}\right)$ & $35,1(4,4)$ & $27,6(4,2)$ & $36,6(3,7)$ & $0,65(0,33)$ \\
AST/ALT & $1,06(0,42)$ & $0,72(0,46)$ & $1,46(0,42)$ & $155,7(51,0)$ \\
Colesterol $(\mathrm{mg} / \mathrm{dL})$ & $156,0(32,0)$ & $163,0(38,0)$ & $137,6(31,7)$ & $162,4(57,0)$ \\
Triglicéridos $(\mathrm{mg} / \mathrm{dL})$ & $167,7(64,2)$ & $183,6(79,6)$ & $130,6(27,3)$ & $332333(172434)$ \\
Plaquetas $\left(\mathrm{mm}^{3}\right)$ & $338438(125712)$ & $366857(244249)$ & $269600(46490)$ & \\
\hline
\end{tabular}

Los datos se representan como media \pm desviación estándar. 
Adicionalmente, los resultados muestran mayor frecuencia de la enfermedad en mujeres residentes de áreas urbanas, datos similares se reportan en otras investigaciones en Colombia (34). Estos hallazgos podrían estar asociados con altos niveles de estrés, disminución de calidad de vida e inadecuados hábitos alimenticios; aspectos que han sido valorados también para explicar el alto riesgo cardiovascular de las mujeres en la misma región (35). Además, observar mayoritariamente en el área urbana estas características, asociadas a NAFLD, es un indicio indirecto de trastorno hepático que siempre ha estado vinculado a estrés y a países desarrollados, más que a la ubicación geográfica. Esto es debido al estilo de vida y de alimentación de la población (1). La población estudiada se caracterizó por patologías como diabetes mellitus, obesidad, hipertensión arterial y combinaciones de estas. Otros investigadores han reportado enfermedades asociadas a NAFLD e incluyen las reportadas en este estudio piloto (2).

La frecuencia relativa de estas patologías asociadas a NAFLD fue similar para todos los grupos, con mayor incidencia en obesidad; este factor es uno de los más influyentes y así es resaltado en un reporte de Japón, que encontró una prevalencia de $25 \%$ aproximado en pacientes con NAFLD (15). Evidentemente varía en diferentes regiones por la cultura y estilos de vida. Aun así, otros investigadores consideran que no siempre todos los obesos desarrollan NAFLD, pero resaltan su importancia cuando se suma a otros factores como diabetes, como evento importante a hígado graso (18). Es de resaltar que la organización mundial de la salud para Latino América reporta la existencia de más del 15\% de la población obesa en Argentina, Colombia, Perú, Paraguay y Uruguay. También otros estudios han resaltado que en Latinoamérica es más susceptible a obesidad y diabetes tipo II, por la tendencia de estilo de vida sedentario y consumo de comidas de alto nivel de grasa (17). Adicionalmente, es necesario destacar que la obesidad está correlacionada con prevalencia y severidad de NAFLD; así lo demuestran recientes estudios americanos, donde la prevalencia de obesidad-severidad NAFLD fue de $23,5 \%$ en hombres y $29,7 \%$ en mujeres (16).

Otro factor de riesgo relacionado, para el desarrollo de enfermedades crónicas del hígado, es la diabetes mellitus. Se estima la prevalencia de NAFLD en 60\%-76\% en esta condición, con lo cual la población estudiada acentúa su probabilidad de NAFLD porque la resistencia a la insulina y el estrés oxidativo tienen un papel muy importante en el desarrollo y progresión de NAFLD, debido a la alta concentración de insulina que puede causar falla para suprimir el flujo de ácidos grasos. Todos estos factores asociados a NAFLD confirman la estricta asociación fisiopatológica entre el desequilibrio del metabolismo de lípidos y glucosa establecidos bioquímicamente $(19,21)$.
Si bien en nuestro estudio el $20 \%$ de la población con posibilidad de riesgo de NAFLD presentó DM, es indudable que el desbalance metabólico de lípidos y carbohidratos que existe es de alta influencia para el establecimiento de NAFLD, con lo que se hace necesario confirmar este diagnóstico. En los resultados, se muestra que los diferentes grupos no presentan un puntaje significativo para fibrosis hepática (datos no mostrados), dato que concuerda con los valores de TG y colesterol. Los TG, se consideran hepatoprotectores en el contexto de NAFLD, protegen de la lipotoxicidad, por proveer un mecanismo de buffer para la acumulación de ácidos grasos libres, pero esto no es inofensivo, ya que la esteatosis se ha asociado con obesidad, dislipidemia, resistencia a insulina e hipertensión. A diferencia de los TG, el colesterol está asociado NASH. Un estudio in vitro en Caenorhabditis elegans demostró que el colesterol libre, más que los triglicéridos y el acumulo de ácidos grasos libres, sensibilizan el hígado a esteatosis hepática mediante factor de necrosis tumoral alfa (FNT- $\alpha$ ) y Fas. También hay evidencias en modelos de ratones hiperlipidémicos, en los que el colesterol libre es un factor de riesgo importante en la transición de esteatosis a NASH (36-40).

Finalmente, los índices De Ritis mayores de 1,0, encontrados en los pacientes y probablemente derivados de la producción y liberación mitocondrial de AST, indican que existe progresión de la fibrosis hepática en los individuos (24). Se ha sugerido que estos cambios en los niveles de transaminasas preceden al hígado graso y se asocian con tendencia a resistencia a la insulina (4). Si bien la relación De Ritis mayor a 1,0 podría indicar el desarrollo de cirrosis, la elevación de los niveles de las transaminasas en hígado graso no siempre es evidencia del grado de afección hepatocelular (5). Por tanto, con los resultados bioquímicos encontrados, es importante realizar un estudio amplio del diagnóstico de NAFLD por imagenología, histología y marcadores bioquímicos y moleculares que permitan elucidar mecanismos moleculares, que subyacen a NAFLD y proveer nuevas bases para el tratamiento de la inflamación hepática.

\section{CONCLUSIÓN}

En resumen, los resultados preliminares de este estudio piloto sugieren que existe una población con alto riesgo de padecer hígado graso a la luz de los factores asociados. Las características de la población y la relación AST/ALT mayor a 1,0, y otras variables bioquímicas, alertan sobre la necesidad de un estudio bioquímico-molecular más completo.

\section{Agradecimientos}

A la Facultad de Medicina de la Universidad de Cartagena, Hospital Universitario del Caribe, Semillero de Investigación 
del Grupo Prometeus, Residentes Especialidad en Cirugía Universidad de Cartagena (Dr. Pedro Vides De la Cruz, Dr. Adolfo Roldan Chica, Dr. Luis Gerardo Villota González, Dr. Rubén Agresott Marsiglia).

\section{Conflicto de intereses}

Los autores declaran no tener ningún conflicto de intereses.

\section{Fuentes de financiación}

Los autores declaran financiación parcial por parte del plan de Fortalecimiento de la Maestría en Bioquímica y Financiación autónoma de parte del grupo reconocido por Colciencias: Prometeus \& Biomedicina aplicada a las Ciencias clínicas.

\section{REFERENCIAS}

1. Marchesini G, Bugianesi E, Forlani G, Cerrelli F, Lenzi M, Manini R, et al. Nonalcoholic fatty liver, steatohepatitis, and the metabolic syndrome. Hepatology (Baltimore, Md). 2003;37(4):917-23.

2. Adams LA, Waters OR, Knuiman MW, Elliott RR, Olynyk JK. NAFLD as a Risk Factor for the Development of Diabetes and the Metabolic Syndrome: An Eleven-Year Follow-up Study. Am J Gastroenterol. 2009;104(4):861-7.

3. ClarkJM. The epidemiology of nonalcoholic fatty liver disease in adults. J Clin Gastroenterol. 2006;40 Suppl 1:S5-10.

4. Onyekwere CA, Ogbera AO, Balogun BO. Non-alcoholic fatty liver disease and the metabolic syndrome in an urban hospital serving an African community. Ann Hepatol. 2011;10(2):119-24.

5. Eguchi Y, Hyogo H, Ono M, Mizuta T, Ono N, Fujimoto $\mathrm{K}$, et al. Prevalence and associated metabolic factors of nonalcoholic fatty liver disease in the general population from 2009 to 2010 in Japan: a multicenter large retrospective study. J Gastroenterol. 2012;47(5):586-95.

6. Amarapurkar D, Kamani P, Patel N, Gupte P, Kumar P, Agal $\mathrm{S}$, et al. Prevalence of non-alcoholic fatty liver disease: population based study. Ann Hepatol. 2007;6(3):161-3.

7. Bellentani S, Saccoccio G, Masutti F, Croce LS, Brandi G, Sasso F, et al. Prevalence of and risk factors for hepatic steatosis in Northern Italy. Ann Intern Med. 2000;132(2):112-7.

8. Bellentani S, Bedogni G, Miglioli L, Tiribelli C. The epidemiology of fatty liver. Eur J Gastroenterol Hepatol. 2004;16(11):1087-93.

9. Caballeria L, Pera G, Auladell MA, Toran P, Munoz L, Miranda $D$, et al. Prevalence and factors associated with the presence of nonalcoholic fatty liver disease in an adult population in Spain. Eur J Gastroenterol Hepatol. 2010;22(1):24-32.

10. Williamson RM, Price JF, Glancy S, Perry E, Nee LD, Hayes $\mathrm{PC}$, et al. Prevalence of and risk factors for hepatic steatosis and nonalcoholic Fatty liver disease in people with type 2 diabetes: the Edinburgh Type 2 Diabetes Study. Diabetes Care. 2011;34(5):1139-44.

11. Browning JD, Szczepaniak LS, Dobbins R, Nuremberg P, Horton JD, Cohen JC, et al. Prevalence of hepatic steatosis in an urban population in the United States: impact of ethnicity. Hepatology (Baltimore, Md). 2004;40(6):1387-95.

12. Castro-Martinez MG, Banderas-Lares DZ, RamirezMartinez JC, Escobedo-de la Pena J. Prevalence of nonalcoholic fatty liver disease in subjects with metabolic syndrome. Cir Cir. 2012;80(2):128-33.

13. Araujo LM, De Oliveira DA, Nunes DS. Liver and biliary ultrasonography in diabetic and non-diabetic obese women. Diabetes Metab. 1998;24(5):458-62.

14. Boza C, Riquelme A, Ibanez L, Duarte I, Norero E, Viviani $\mathrm{P}$, et al. Predictors of nonalcoholic steatohepatitis (NASH) in obese patients undergoing gastric bypass. Obes Surg. 2005;15(8):1148-53.

15. Chavez-Tapia NC, Sanchez-Avila F, Vasquez-Fernandez F, Torres-Machorro A, Tellez-Avila FI, Uribe M. Nonalcoholic fatty-liver disease in pediatric populations. Journal of pediatric endocrinology \& metabolism. JPEM. 2007;20(10):1059-73.

16. Feng RN, Du SS, Wang C, Li YC, Liu LY, Guo FC, et al. Lean-non-alcoholic fatty liver disease increases risk for metabolic disorders in a normal weight Chinese population. World journal of gastroenterology. 2014;20(47):17932-40.

17. Seo HI, Cho YK, Lee WY, Rhee EJ, Sung KC, Kim BS, et al. Which metabolic syndrome criteria best predict the presence of non-alcoholic fatty liver disease? Diabetes Res Clin Pract. 2012;95(1):19-24.

18. Sun L, Lu SZ. Association between non-alcoholic fatty liver disease and coronary artery disease severity. Chin Med J (Engl). 2011;124(6):867-72.

19. De Alwis NM, Day CP. Non-alcoholic fatty liver disease: the mist gradually clears. Journal of hepatology. 2008;48(Suppl 1):S104-12.

20. Yoon KH, Lee JH, Kim JW, Cho JH, Choi YH, Ko SH, et al. Epidemic obesity and type 2 diabetes in Asia. Lancet (London, England). 2006;368(9548):1681-8.

21. Otgonsuren M, Stepanova M, Gerber L, Younossi ZM. Anthropometric and clinical factors associated with mortality in subjects with nonalcoholic fatty liver disease. Dig Dis Sci. 2013;58(4):1132-40.

22. Romeo S, Kozlitina J, Xing C, Pertsemlidis A, Cox D, Pennacchio LA, et al. Genetic variation in PNPLA3 confers susceptibility to nonalcoholic fatty liver disease. Nature genetics. 2008;40(12):1461-5.

23. Kawaguchi T, Sumida Y, Umemura A, Matsuo K, Takahashi M, Takamura T, et al. Genetic polymorphisms of the human PNPLA3 gene are strongly associated with severity of non-alcoholic fatty liver disease in Japanese. PloS one. 2012;7(6):e38322.

24. Daliri MR. A hybrid automatic system for the diagnosis of lung cancer based on genetic algorithm and fuzzy extreme learning machines. J Med Syst. 2012;36(2):1001-5. 
25. Kleine FD. The diagnostic significance of the De-Ritis quotient. Deutsche Zeitschrift fur Verdauungs- und Stoffwechselkrankheiten. 1966;25(6):355-62.

26. Rej R. Aspartate aminotransferase activity and isoenzyme proportions in human liver tissues. Clin Chem. 1978;24(11):1971-9.

27. Botros M, Sikaris KA. The de ritis ratio: the test of time. The Clinical biochemist Reviews / Australian Association of Clinical Biochemists. 2013;34(3):117-30.

28. Williams CD, Stengel J, Asike MI, Torres DM, Shaw $\mathrm{J}$, Contreras $\mathrm{M}$, et al. Prevalence of nonalcoholic fatty liver disease and nonalcoholic steatohepatitis among a largely middle-aged population utilizing ultrasound and liver biopsy: a prospective study. Gastroenterology. 2011;140(1):124-31.

29. Vernon G, Baranova A, Younossi ZM. Systematic review: the epidemiology and natural history of non-alcoholic fatty liver disease and non-alcoholic steatohepatitis in adults. Aliment Pharmacol Ther. 2011;34(3):274-85.

30. Lonardo A, Bellentani S, Argo CK, Ballestri S, Byrne CD, Caldwell SH, et al. Epidemiological modifiers of non-alcoholic fatty liver disease: Focus on high-risk groups. Digestive and liver disease: official journal of the Italian Society of Gastroenterology and the Italian Association for the Study of the Liver. 2015.

31. Weston SR, Leyden W, Murphy R, Bass NM, Bell BP, Manos $\mathrm{MM}$, et al. Racial and ethnic distribution of nonalcoholic fatty liver in persons with newly diagnosed chronic liver disease. Hepatology (Baltimore, Md). 2005;41(2):372-9.

32. Caldwell SH, Harris DM, Patrie JT, Hespenheide EE. Is NASH underdiagnosed among African Americans? Am J Gastroenterol. 2002;97(6):1496-500.
33. Watanabe $S$, Hashimoto $E$, Ikejima $K$, Uto $H$, Ono $M$, Sumida Y, et al. Evidence-based clinical practice guidelines for nonalcoholic fatty liver disease/nonalcoholic steatohepatitis. J Gastroenterol. 2015;50(4):364-77.

34. Vanegas R CM, Restrepo R C, Vargas G N, Marín C AE, Martínez S LM, Yepes D CE, et al. Characterization of Patients with Non-Alcoholic fatty Liver Disease in a Highly Complex Colombian Hospital in 2013. Rev Col Gastroenterol. 2014;29(4):342-6.

35. Cabarcas-Barboza O, Pulgar-Emiliani MI, Alvear-Sedan C. Cardiovascular risk factors in young adult people of cartagena de indias. Colombia. Revista Ciencias Biomedicas. 2014;5(2):211-9.

36. Puri P, Baillie RA, Wiest MM, Mirshahi F, Choudhury $\mathrm{J}$, Cheung $\mathrm{O}$, et al. A lipidomic analysis of nonalcoholic fatty liver disease. Hepatology (Baltimore, Md). 2007;46(4):1081-90.

37. Yamaguchi K, Yang L, McCall S, Huang J, Yu XX, Pandey SK, et al. Inhibiting triglyceride synthesis improves hepatic steatosis but exacerbates liver damage and fibrosis in obese mice with nonalcoholic steatohepatitis. Hepatology (Baltimore, Md). 2007;45(6):1366-74.

38. Asrih M, Jornayvaz FR. Inflammation as a potential link between nonalcoholic fatty liver disease and insulin resistance. The Journal of endocrinology. 2013;218(3):R25-36.

39. Caballero F, Fernandez A, De Lacy AM, Fernandez-Checa JC, Caballeria J, Garcia-Ruiz C. Enhanced free cholesterol, SREBP-2 and StAR expression in human NASH. Journal of hepatology. 2009;50(4):789-96.

40. Walenbergh SM, Shiri-Sverdlov R. Cholesterol is a significant risk factor for non-alcoholic steatohepatitis. Expert review of gastroenterology \& hepatology. 2015;9(11):1343-6. 